\title{
Direct Measurement of the Surface Energy of
}

\section{Graphene}

Christian D. van Engers ${ }^{1,2}$, Nico E.A. Cousens ${ }^{1}$, Vitaliy Babenko ${ }^{2,3}$, Jude Britton ${ }^{2}$, Bruno Zappone $^{4}$, Nicole Grobert ${ }^{2}$ and Susan Perkin ${ }^{1 *}$

1) Department of Chemistry, University of Oxford, South Parks Road, Oxford OX1 3QZ, United Kingdom

2) Department of Materials, University of Oxford, Parks Road, Oxford OX1 3PH, United Kingdom

3) Centre for Advanced Photonics and Electronics, Cambridge University, 9 JJ Thomson Ave, Cambridge, CB3 0FA, United Kingdom.

4) Consiglio Nazionale delle Ricerche, Istituto di Nanotecnologia (CNR-Nanotec), c/o Department of Physics, Università della Calabria, Rende (CS) 87036, Italy

*E-mail: susan.perkin@chem.ox.ac.uk

KEYWORDS: Surface Force Balance, Surface Force Apparatus, Graphene, Surface Energy, Adhesion Energy, Energy of Cohesion 


\section{ABSTRACT}

Graphene produced by chemical vapor deposition (CVD) is a promising candidate for implementing graphene in a range of technologies. In most device configurations, one side of the graphene is supported by a solid substrate, while the other side is in contact with a medium of interest, such as a liquid or other 2-dimensional material within a van der Waals stack. In such devices, graphene interacts on both faces via non-covalent interactions, and therefore surface energies are key parameters for device fabrication and operation. In this work, we directly measured adhesive forces and surface energies of CVD-grown graphene in dry nitrogen, water and sodium cholate using a modified surface force balance. For this we fabricated large $\left(\sim 1 \mathrm{~cm}^{2}\right)$ and clean graphene-coated surfaces with smooth topography at both macro- and nano-scales. By bringing two such surfaces into contact and measuring the force required to separate them, we measured the surface energy of single-layer graphene in dry nitrogen to be $115 \pm 4 \mathrm{~mJ} / \mathrm{m}^{2}$, which was similar to that of few-layer graphene $\left(119 \pm 3 \mathrm{~mJ} / \mathrm{m}^{2}\right)$. In water and sodium cholate, we measured interfacial energies of $83 \pm 7 \mathrm{~mJ} / \mathrm{m}^{2}$ and $29 \pm 6 \mathrm{~mJ} / \mathrm{m}^{2}$, respectively. Our work provides the first direct measurement of graphene surface energy, and is expected to have an impact both on the development of graphene-based devices and contribute to the fundamental understanding of surface interactions. 


\section{TEXT}

\section{Introduction}

Graphene is a material of significant scientific and technological interest due to its outstanding electrical and mechanical performances ${ }^{1}$, impermeability to liquids and gasses ${ }^{2}$, optical transparency ${ }^{3,4}$, high specific surface area and stability ${ }^{5}$. The exploitation of these properties requires graphene to be at the interface between two media, for example when used as a membrane, or between a medium and a supporting solid substrate (e.g. $\mathrm{SiO}_{2}$, hexagonal boron nitride). Therefore, understanding and controlling the interfacial energies of graphene is of paramount importance for the fabrication and operation of graphene-based devices ${ }^{6}$.

While the surface energy of graphite has been investigated as a model for London - van der Waals surface interactions ${ }^{7-10}$, very few studies have focused on the surface energy of graphene. Recently, investigations into the fundamental properties and potential applications of graphene have renewed this interest ${ }^{11-14}$, including computational efforts to determine the interlayer adhesion in van der Waals solids ${ }^{15-18}$. Peng et al. ${ }^{19}$ and Wang et al.$^{20}$ have experimentally determined the adhesion energy of graphene to graphite, by pushing down a graphene beam onto an underlying graphite substrate and by studying the intercalation of noble gas atoms between graphene and a graphite substrate, respectively measuring $154 \pm 21 \mathrm{~mJ} / \mathrm{m}^{2}$ and $110 \pm 5 \mathrm{~mJ} / \mathrm{m}^{2}$ as interfacial energies. Contact angle and finite-dilution inverse gas chromatography measurements have yielded a range of (lower) surface energy values ${ }^{21}$. However, these measurements may be influenced by the adsorption of airborne contaminants and, in case of contact angle measurements, both the method of measurement and the underlying substrate $^{22,23}$. In particular, calculation of surface energies from contact angle measurements rely 
upon models such as OWRK (after Owens, Wendt, Rabel and Kaelble), which implicitly assume separability and additivity of polar and dispersive contributions $\mathrm{s}^{24,25}$. Theoretical work has predicted values in the range $144-171 \mathrm{~mJ} / \mathrm{m}^{2} 15-18$.

In this study, we use a direct method to measure the surface energy of graphene synthesized using chemical vapor deposition (CVD) techniques, commonly proposed for largescale production of high-quality graphene ${ }^{26}$. We use the term 'direct', since the experiments consist of two macroscopic graphenes pulled apart from adhesive contact. The graphenes are mounted on two cylindrical lenses in crossed-cylinder orientation - geometrically equivalent to a sphere in contact with a flat surface - for which the contact mechanics is well known, allowing reliable interpretation of the measured pull-off force. To convert the measured pull-off force into surface energy, the contact can be described using JKR-theory (after Johnson, Kendall and Roberts $^{27}$, as has been shown by others to apply to this setup ${ }^{28,29}$.

Due to the use of catalytic metal substrates for the CVD synthesis, a transfer from the growth substrate to the target substrate of interest is required. We have previously developed a clean transfer method in which the graphene interface to be probed is put in contact with only a $\mathrm{Cu}$-etching solution and ultrapure water in a particle-free atmosphere ${ }^{30}$. Therefore, we measured graphene surface energies in conditions close to the actual environment of (graphene-based) micro-devices fabrication (e.g. clean room), rather than the surface energy of single crystal graphene in vacuum.

The apparatus used for measurement of the graphene-graphene pull-apart force, and hence surface energy, is a surface force balance (SFB, also known as surface force apparatus) $)^{31}$. SFBs and other surface force apparatuses have been extensively used for determining 
adhesion/cohesion forces, surface/interface energies and Hamaker constants for a large variety of materials and surfaces, including graphite, both in gases and liquids ${ }^{32}$. In the present work, cylindrical glass lenses (radius, $\mathrm{R}_{\mathrm{L}}=1 \mathrm{~cm}$ ) were first coated with a mirror layer of $\mathrm{Au}(40 \mathrm{~nm}$ thickness) that enabled the measurement of separation distance between two such lenses via multiple-beam interferometry ${ }^{30}$. Subsequently the Au-coated lenses were covered with an ultrasmooth and thin layer of hard epoxy (elastic modulus $3.4 \mathrm{GPa}^{28}$ ), upon which the graphene sheets (area $\sim 1 \mathrm{~cm}^{2}$ ) were adhered. The graphene-coated cylindrical lenses, mounted with axes at $90^{\circ}$ (crossed-cylinder geometry, equivalent to a sphere approaching a flat surface) were brought into contact (fig. 1) in air or liquid. They were then slowly pulled apart against the deflection of a spring with known spring constant $k_{\text {spring }}$. When the restoring elastic force of the spring was equal to the adhesive (cohesive) force $F_{a d h}$, the surfaces suddenly jumped out from contact to a new rest position, travelling a distance $d_{j u m p}$.

The pull-off force was calculated using Hooke's law: $F_{\text {adh }}=k_{\text {spring }} \cdot d_{j u m p}$. As long as the surfaces are pulled apart at reversibly slow speeds, the pull-off force $F_{\text {adh }}$ can be directly related to the surface energy $\gamma$ via JKR theory ${ }^{27,29}$, using:

$$
\gamma=\frac{F_{a d h}}{3 \pi R_{G}}
$$

Where $\mathrm{R}_{\mathrm{G}}$ is the radius of curvature of the graphene-coated surfaces at the point of contact. Note that the radius $R_{G}$ of the graphenes may be slightly different from the radius $R_{L}$ of the underlying glass lenses due to small inhomogeneities in thickness of the epoxy glue layer. This difference is measured using secondary interferometry within the SFB, so that the precise value of $R_{G}$ can be used for our calculations. 
Using this direct graphene-graphene pull-apart experiment, with well characterized contact geometry, contact mechanics and graphene that is smooth on a macro- and nanoscopic scale, we find that the surface energy of single layer graphene (SLG) is $\gamma=115 \pm 4 \mathrm{~mJ} / \mathrm{m}^{2}$. For few layer graphene (FLG) we find $\gamma=119 \pm 3 \mathrm{~mJ} / \mathrm{m}^{2}$. We have also measured interfacial energies between FLG and water and FLG and sodium cholate $(\mathrm{NaCh})$, finding $\gamma=83 \pm 7 \mathrm{~mJ} / \mathrm{m}^{2}$ and $\gamma=29 \pm 6 \mathrm{~mJ} / \mathrm{m}^{2}$.

Our results will contribute to the physical understanding and mechanical characterization of graphene as pertinent to a multitude of current graphene technologies. 


\section{Surface preparation}

Graphene-coated surfaces were fabricated using a modified version of a previously reported procedure ${ }^{30}$, designed to produce clean, large-area $\left(\sim 1 \mathrm{~cm}^{2}\right)$, single-layer (SLG) or fewlayers (FLG) graphene with very small nanoscale roughness ( $1 \mathrm{~nm}$ RMS $)$, glued onto hemicylindrical glass lenses covered with a template-stripped layer of epoxy layer with minimum thickness inhomogeneity, ensuring a correct determination of surface energies within a singlepoint contact geometry with $R_{G} \approx R_{L}$. Here we briefly outline the procedure; further extensive details and characterization of the graphene substrates can be found in the Supplementary Information (SI).

Graphene was first grown by CVD on polycrystalline $\mathrm{Cu}$ substrate to cover more than $99 \%$ of the $\mathrm{Cu}$ substrate. After synthesis, the quality of the as-grown graphene was verified using Raman, electron and optical microscopy (see SI, fig. S2-S6). In order to transfer the graphene onto the lenses (fig. S6), first a layer of polystyrene (PS) was spin-coated onto graphene as a polymer support. Then, the $\mathrm{Cu}$ substrate was etched using ammonium persulphate. The graphene-PS assembly was rinsed in ultra-pure water and placed onto a piece of freshly cleaved muscovite mica (supported on a thick rigid mica plate) so that the graphene side of interest faced smooth, contamination free mica surface ${ }^{33}$. The PS support was then dissolved in toluene before gluing the graphene sheet onto the Au-coated cylindrical glass lenses of the SFB (fig. S1, steps 1A-D).

In order to glue the graphene onto the Au-coated cylindrical lens, a film of epoxy was fabricated separately and then deposited on the Au-coated surfaces to minimize roughness and inhomogeneity. The epoxy film was prepared by spin-coating from chloroform solution onto a 
thin flexible piece of freshly cleaved muscovite mica (supported on a thick rigid mica plate). This mica/epoxy assembly was then placed onto the Au-coated lens with the epoxy side down and heated to the glass transition temperature of the epoxy. After cooling down, the mica sheet was stripped off in ultrapure water (fig. S1, steps 2A-C). Acting as a template, the mica sheet left an epoxy layer with uniform thickness and very smooth topography, to be glued to graphene. After drying and annealing the epoxy, the graphene/mica assembly is placed onto the epoxy/SFB lens surface (graphene side down). Subsequently the mica was stripped off in ultra-pure water, exposing a smooth graphene surface (fig. S1, steps 3,4). During the lasts steps of the preparation, the graphene covered SFB lens was exposed to particle-free ambient atmosphere for no longer than 10 minutes. The graphene-covered SFB lens was then loaded into the SFB chamber, which was purged with $\mathrm{N}_{2}$.

The thickness $h$ of the epoxy layer was typically $2-4 \mu \mathrm{m}$. Uniformity of the layer thickness was examined using optical profilometry (fig. S8) and by observing the Newton's rings produced by the surface when viewed with a reflection optical microscope through a bandpass filter (fig. 1d). The glue thickness deviated by less than $1 \mu \mathrm{m}$ over a distance of $1 \mathrm{~mm}$. At the nanoscale, we measured a RMS roughness of the graphene surface of $\sim 1 \mathrm{~nm}$ using atomic force microscopy as previously reported ${ }^{30}$ (fig. 1c). 


\section{Interferometry}

The reflective Au coatings on the crossed cylindrical lenses of the SFB were used to implement a multiple-beam Fabry-Pérot interferometer with curved mirror geometry in order to determine both surface separation distance and radius of curvature ${ }^{30}$ (fig. 2). Light waves from a collimated white light source underwent multiple reflections within the interferometer and were selectively transmitted at wavelengths corresponding to constructive interference. These waves were directed through a narrow slit into an imaging spectrometer and the resulting spectrogram, showing interference fringes, was recorded by a CCD camera. The slit could be oriented parallel to either one of the cylinder axes ( $x$ or $y$ ). Sharp primary fringes, corresponding to fringes of equal chromatic order (FECO), were produced by multiple reflections between Au mirrors and appeared curved due to the increasing separation between the mirrors moving along the slit direction from the contact position (fig. $2 \mathrm{a}, \mathrm{b}$; green solid line). Secondary fringes were also observed due to multiple reflections within the epoxy layers, i.e. reflections at the epoxy/Au and epoxy-graphene/air interfaces (fig. 2a,b; dashed and dotted line) ${ }^{34}$.

Whilst the curvature of the primary fringes as viewed in the spectrogram reveals the curvature $R_{L}$ of the Au mirrors, it does not provide an exact measure of the radius $R_{G}$ of curvature of the graphene surfaces due to the non-uniform thickness of the epoxy layer (fig. 1b). To account for the difference, we also analyzed the secondary fringes (fig. 3a). Two sets of secondary fringes were observed. The apex of the secondary fringes was aligned with the apex of the primary fringes and had a parabolic shape with radius $R_{S}$. One set had very large radius, $R_{S / /}$ (horizontal band in fig. 3a), and was produced by the surface whose cylinder axes was parallel to the spectrometer slit ( $y$-axis of the bottom surface in fig. $2 \mathrm{a}$ ). The value of $R_{S / /}$ reflects the slight random non-uniformity of the epoxy thickness layer over large lateral distances in $y\left(\mathrm{~T}_{2}\right.$, fig. 2c), 
in agreement with fig. $1 \mathrm{~d}$. The other set of secondary fringes showed a much smaller radius, $R_{S}$ (parabolic bands in figure 3a), and was produced by the epoxy layer on the surface aligned perpendicular to the slit (top surface in fig. 2a). Indeed, the epoxy layer on this surface had a net curvature along the slit direction (variation of $\mathrm{T}_{1}$ in fig. 2c). The secondary fringe radius $R_{S}$ can be related to the radius of curvature $R_{G}$ of the graphene axis along the slit direction using the formula (see Supplementary Information, section 3):

$$
R_{G^{\perp}}=\frac{R_{S^{\perp}} R_{L}}{R_{L^{\prime}}+R_{S^{\perp}}}
$$

Note that if the epoxy is of precisely uniform thickness (i.e. the secondary fringes appear as horizontal lines in the spectrogram) $R_{S^{\perp}} \rightarrow \infty$ and $R_{G^{\perp}} \approx R_{L}$. To obtain the radius of curvature $R_{G}$ of the other lens, the image of the surfaces entering slit was rotated by $90^{\circ}$ using a dove prism. The effective radius of curvature $R_{G}$ used in equation 1 is the geometric mean of the two $R_{G}$ values. Implementing the above procedure to determine the graphene radius of curvature was necessary for achieving accurate surface energies. Further, we note that the fabrication method used here results in a very uniform epoxy layer thickness, such that the radius of curvature of the graphene surfaces was very close to that of the underlying glass lenses: $0.9<R_{L} / R_{G}<1.1$

The interplay between the multiple reflections within our interferometer is complex. To validate our interpretation of the fringe origins and resulting fringes patterns, we extracted the value for $R_{S}$ from a real interferogram and used it as input for a simulated interferogram using multi-layer calculations based on transfer matrices (fig. 3). For the refractive index of graphene, we chose $\mathrm{RI}=2.6+1.3 \mathrm{i}^{35}$. The optical constants for Au were taken from McPeak et al..$^{36}$ and the refractive index of the epoxy $(n=1.593)$ was measured using ellipsometry and reflectometry. 
Figure $3 \mathrm{~b}$ is a diagram showing the main input parameters $R_{G}, R_{L}, T_{1}, T_{2}$ (the thicknesses of the two epoxy layers on each surface) and $T_{3}$, the separation between the surfaces for the model used. The thickness $T_{1}$, and $T_{3}$, varied as a function of the y-coordinate to account for the thickness variation of the epoxy. The gold mirror and graphene layer were not included in the schematic for clarity. Fig. 3a shows the experimental interferogram with the secondary fringes corresponding to $R_{S}$ visible as two arcs, superimposed on the primary fringes, and the fringe corresponding to $R_{S / /}$ shows as a bright horizontal modulation across the lower arc. Using $R_{S}=$ $210 \mathrm{~mm}\left(\mathrm{R}_{\mathrm{G}}=10.5 \mathrm{~mm}\right)$ as extracted from the experimental data (fig. 3a), we find excellent agreement between the measured and calculated interferograms (fig. 3c). The differences between calculation and experiment mainly arise from the approximation of the graphene surface as a sphere, the difficulty in determining the exact location and shape of the secondary fringes from the experimental spectra, and imperfections in the experimental spectra (e.g. roughness of the evaporated $\mathrm{Au}$ mirror). Analysis of the calculated spectrum demonstrates that our interpretation of the complex fringe pattern is appropriate and validates equation 2 .

As such, the measurement of $R_{G}$ is obtained from equation 2 after determining the radii $R_{S}$ of the top and bottom glue layers to calculate the value of $R_{G}$. The surface energy can thus be determined via the JKR equation (eq. 1). In this way, we measured the adhesion force and surface/interfacial energies of the macroscopic smooth graphene surface in dry $\mathrm{N}_{2}$, water and sodium cholate solution. 


\section{Results \& Discussion}

Fig. 4 shows the results of our direct measurement of the surface energy of SLG and FLG graphene in dry nitrogen atmosphere. The surface energy of the underlying epoxy alone and the interfacial energies for graphene in water and aqueous solution of sodium cholate are also shown for comparison. Sodium cholate was chosen because it is a surfactant used as intercalant in the solvo-mechanical exfoliation of graphite to produce graphene ${ }^{37}$. The surface energies of FLG and SLG were found to be $\gamma_{\mathrm{FLG}}=119 \pm 3 \mathrm{~mJ} / \mathrm{m}^{2}$ and $\gamma_{\mathrm{SLG}}=115 \pm 4 \mathrm{~mJ} / \mathrm{m}^{2}$. These values represent the first direct measurements of the surface energy of both few and single layer graphene. For consistency, each data point in the histogram in fig. 4 represents a measurement at a different location on the graphene-covered SFB lenses. Experiments were performed with different pairs of graphene-covered lenses. For FLG, eight different measurements were conducted using two different pairs of lenses; for SLG, it includes 11 different measurements over two different pairs of lenses.

We verified the presence of graphene in situ by probing the electrical conductivity between the surfaces in contact, to ensure we did not measure forces resulting from mica that had not been removed during the surface preparation. Since both mica and the underlying epoxy are insulators, the conductive behavior provides strong evidence that graphene is indeed present on both lenses. Further, the surface energies of FLG and SLG glued on the epoxy layers were significantly higher than the surface energy of bare epoxy, $\gamma_{\text {epoxy }}=79 \pm 7 \mathrm{~mJ} / \mathrm{m}^{2}$. This calibration directly shows the substantial contribution to the adhesion force and surface energy due to the atomic layer (or layers) of graphene. We note that interactions between the polymer chains of the 
epoxy may influence the value measured for the surface energy of the epoxy, but do not influence the value measured for graphene ${ }^{38}$.

By separating the graphenes whilst fully immersed in water, the interfacial energy of graphene in water was found to be $\gamma_{\text {graphene-water }}=83 \pm 7 \mathrm{~mJ} / \mathrm{m}^{2}$ (fig. $3 \mathrm{c}$ ). This value was compared to the one obtained from the measurement of the static contact angle of water $(\theta)$ on graphene surfaces, with samples prepared according to the same procedure, but on a planar substrate. The resulting static water contact angle (WCA) values were $71 \pm 1^{\circ}$ for SLG and $70 \pm$ $1^{\circ}$ for FLG. From the measured values of $\theta$ and the interfacial energy of graphene in water,

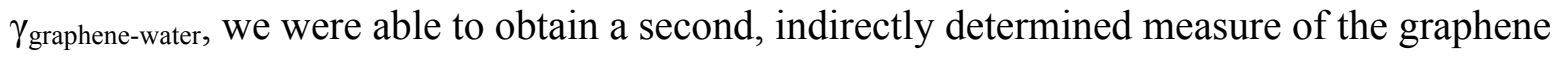
surface energy $\gamma_{\text {graphene }}$ using the Young-Dupré equation (see SI, section 5). The resulting value was $\gamma_{\text {graphene }}=106 \pm 7 \mathrm{~mJ} / \mathrm{m}^{2}$; comparable but a slightly lower than the values $\gamma_{\mathrm{FLG}}$ and $\gamma_{\mathrm{SLG}}$ obtained from adhesion force measurements (fig. 4c). This result highlights the importance of directly measuring graphene surface energy as in our graphene-SFB method.

The absorption of airborne contaminants on pristine surfaces is a well-known phenomenon that has recently been under rigorous investigation with respect to its effect on the WCA on graphite. A recent examination of the published body of literature by Kozbial et al., indicates that the static WCA on pristine graphitic surfaces is $\theta \approx 65^{\circ}$. Upon exposure to laboratory air, this value increases to $\theta \approx 90^{\circ}$. This further demonstrates the cleanliness of our surface preparation ${ }^{23}$.

When immersed in aqueous sodium cholate solution $(\mathrm{NaCh})$, the interfacial energy of graphene was significantly lowered with respect to the graphene surface energy, to $\gamma_{\mathrm{NaCh}}=29 \pm 6$ 
$\mathrm{mJ} / \mathrm{m}^{2}$. Indeed, sodium cholate is a surfactant that is known to stabilize graphene in solution by the absorption of a monolayer onto the graphene surface ${ }^{39}$.

It was found that the majority of the pull-off measurements on the graphene surfaces, both in nitrogen or liquid, was not reversible and yielded decreasing values of the surface or interfacial energy on subsequent measurements at the same location on the SFB lenses. In contrast, the pull off was reversible (over more than five measurements) for the bare (epoxycoated) SFB lenses. This points to a tendency of the graphene to break or delaminate during pull off. The surface instability may be linked to the relative weak bonds created in the plane of the graphene sheet at the boundaries between crystalline domains of CVD-graphene ${ }^{40}$, which can be easily broken during pull-off, leading to tearing, delamination or exfoliation. The grain sizes of the few layer graphene and single layer graphene are $\sim 5-10 \mu \mathrm{m}$ and $\sim 50-100 \mu \mathrm{m}$ respectively, which is comparable but smaller in size to the contact diameter in our experiment predicted from JKR-theory $(\mathrm{d} \approx 100 \mu \mathrm{m})$ (see fig. S2, S4 and Supplementary Information, section 4). Recent work has shown that two-dimensional solids exhibit a unique behavior in which the surface energy of graphene is sufficient to promote self-tearing, sliding and folding of the graphene sheets, in particular in presence of lateral forces ${ }^{41}$. It is thus possible that the lowered adhesion upon subsequent pull offs originates from tearing, sliding or folding due to lateral distortions and vibrations and the presence of defects (e.g. grain boundaries) within the contact area. In future experiments, it may be possible to carry out experiments with grain sizes substantially larger than the $100 \mu \mathrm{m}$ contact area which will help to reduce the influence of these factors. 
It is striking that the presence of a single layer of graphene was able to substantially increase the cohesive force and surface energy of the graphene-epoxy-coated surface with respect to the bare epoxy-coated surface. For the epoxy, a non-conducting polymer, adhesion is due to the van der Waals interactions, which arise from correlated charge fluctuations in the molecular structure and are proportional to the (modest) polarizability of the polymer. In the case of graphene, consisting of a sp²-hybridized layer with delocalized electron density in pi-orbitals above and below the plane, the correlated charge fluctuations occurring when two graphene surfaces approach face-to-face, are expected to be much larger and thus lead to stronger adhesion. In general, the van der Waals forces between two bodies at small separation (e.g. in contact), covered with a surface film, is dominated by the interaction between the surface layers. Therefore, the presence of the graphene film governs the adhesion ${ }^{32}$.

The surface energy values in this work measured for graphene, lie close to those for the interfacial energy between graphite and graphene measured by Peng et al..$^{19}\left(154 \pm 21 \mathrm{~mJ} / \mathrm{m}^{2}\right)$ and Wang, J. et al. ${ }^{20}\left(110 \pm 5 \mathrm{~mJ} / \mathrm{m}^{2}\right)$. For graphite, which has been more intensively studied, Koren et $a l .{ }^{12}$ and Wang et al. ${ }^{13}$ recently measured the surface energy of graphite mesas through manipulation of the mesa, finding $\gamma=113 \pm 3 \mathrm{~mJ} / \mathrm{m}^{2}$ and $\gamma=185 \pm 10 \mathrm{~mJ} / \mathrm{m}^{2}$. Earlier experiments have yielded values within the range of these two experiments through $e . g$. heat of wetting data $\left(130 \mathrm{~mJ} / \mathrm{m}^{2}\right)^{7}$, examination of nanotube collapse $\left(107 \pm 38 \mathrm{~mJ} / \mathrm{m}^{2}\right)^{9}$ and desorption measurements $\left(186 \pm 15 \mathrm{~mJ} / \mathrm{m}^{2}\right)^{10}$.

Our measurements use CVD-graphene and are therefore relevant to use of graphene in high-end electronics. CVD-graphene requires the use of a transfer step to transfer the graphene from the growth substrate onto the target substrate, during which - in our experiments - the surface is exposed to etchant, ultrapure water and particle-free laboratory air. As such, the 
absorption of air-borne contaminants or even the presence of an adsorbed layer of water ${ }^{42}$ may affect the measured surface energy in a manner similar to that in ultra-clean electronics fabrication facilities. Further, incommensurability of the two graphenes that are brought into contact would be expected to lower the effectively measured surface energy. The effect of incommensurability on the surface energy of graphite has been estimated to be in the range of 10 $\mathrm{mJ} / \mathrm{m}^{2}$ by Wang et al. ${ }^{13}$. In our case, the contact area is larger or equal in size to average grain size the CVD-grown single and few layer graphene. As such, the measured adhesion can be considered an average over different twist angles existing between incommensurate graphene sheets. Finally, the roughness of our samples may contribute to measuring a lower value of the effective surface energy by reducing the effective area of contact. As a comparison, the RMS roughness measured $(\sim 1 \mathrm{~nm})$ is large compared to that of a HOPG terrace, which is atomically smooth $(\mathrm{RMS} \sim 0.03 \mathrm{~nm})^{43}$, but of similar magnitude to the stepped surface of cleaved HOPG $(\operatorname{RMS} 0.7 \mathrm{~nm}, \sim 2 \mathrm{~nm})^{44,45}$.

\section{Conclusion}

In conclusion, our experiments represent the first direct measurement of the surface energy of CVD graphene using both single and few layer graphene at $115 \pm 4 \mathrm{~mJ} / \mathrm{m}^{2}$ and $119 \pm 3$ $\mathrm{mJ} / \mathrm{m}^{2}$ respectively. The technique may be extended to other $2 \mathrm{D}$-materials, and allows for measurements in liquids, as we have demonstrated here for water and sodium cholate, of direct importance for the design and fabrication of devices incorporating 2D-materials. Furthermore, the new methodology presented here opens up the possibility of exploring surface forces between graphene sheets across liquids and with applied surface electric potential. 


\section{ASSOCIATED CONTENT}

\section{Supporting Information}

The following files are available free of charge:

SupplementaryInformation.pdf Detailed description of graphene growth and transfer, including analysis; optical profiles of lens surfaces; derivation of equation 2; calculation of contact diameter based on JKR-theory; contact angle measurements

\section{AUTHOR INFORMATION}

\section{Corresponding Author}

* E-mail: susan.perkin@chem.ox.ac.uk

\section{Present address}

Department of Chemistry, University of Oxford, South Parks Road, Oxford OX1 3QZ, United Kingdom

\section{Author Contributions}

The manuscript was written through contributions of all authors. All authors have given approval to the final version of the manuscript. NG \& SP conceived the project. JB \& NC developed the original transfer procedure. CvE further developed the transfer procedure. VB \& CvE performed the graphene growth and characterization. CvE \& NC performed the measurements \& analyses. SP, BZ \& CvE wrote the paper. All the authors provided comments on the text.

\section{Notes}

The authors declare no competing financial interest. 


\section{ACKNOWLEDGEMENTS}

This work was supported by the European Research Council (ERC-2015-StG-676861; ERC2009-StG-240500; ERC-2011-PoC-309786; ERC-2015-PoC-680559), the Leverhulme Trust (RPG-2015-328), The Royal Society UK (International Exchanges), COST MP1303 and the Engineering and Physical Sciences Research Council. We thank the Taiho Kogyo Tribology Research Foundation for a Research Grant. CDvE acknowledges financial support

from Zvi and Ofra Meitar Magdalen Graduate Scholarship. We gratefully acknowledge the following for assistance: R. Jacobs, R. Chakalova, S. Coles, A.M. Smith, E. Milnes-Smith, F. Hausen and M. Balabajew. 


\section{REFERENCES}

(1) Novoselov, K. S.; Geim, A. K.; Morozov, S. V; Jiang, D.; Zhang, Y.; Dubonos, S. V; Grigorieva, I. V; Firsov, A. A. Science 2004, 306 (5696), 666-669.

(2) Bunch, J. S.; Verbridge, S. S. S.; Alden, J. S.; Van Der Zande, A. M.; Parpia, J. M.; Craighead, H. G.; McEuen, P. L. Nano Lett. 2008, 8 (8), 2458-2462.

(3) Wang, X.; Zhi, L.; Müllen, K. Nano Lett. 2008, 8, 323-327.

(4) Bae, S.; Kim, H.; Lee, Y.; Xu, X.; Park, J.-S.; Zheng, Y.; Balakrishnan, J.; Lei, T.; Ri Kim, H.; Song, Y. Il; Kim, Y.-J.; Kim, K. S.; Özyilmaz, B.; Ahn, J.-H.; Hong, B. H.; Iijima, S. Nat. Nanotechnol. 2010, 5 (8), 574-578.

(5) Simon, P.; Gogotsi, Y. Acc. Chem. Res. 2013, 46 (5), 1094-1103.

(6) Koenig, S. P.; Boddeti, N. G.; Dunn, M. L.; Bunch, J. S. Nat. Nanotechnol. 2011, 6 (9), $543-546$.

(7) Girifalco, L. A.; Lad, R. A. J. Chem. Phys. 1956, 25 (4), 693.

(8) Schrader, M. E. J. Phys. Chem. 1980, 84, 2774.

(9) Benedict, L. X.; Chopra, N. G.; Cohen, M. L.; Zettl, A.; Louie, S. G.; Crespi, V. H. Chem. Phys. Lett. 1998, 286 (5), 490-496.

(10) Zacharia, R.; Ulbricht, H.; Hertel, T. Phys. Rev. B 2004, 69 (15), 1-7.

(11) Gould, T.; Liu, Z.; Liu, J. Z.; Dobson, J. F.; Zheng, Q.; Lebègue, S. J. Chem. Phys. 2013, $139(22), 224704$.

(12) Koren, E.; Rawlings, C.; Knoll, A. W.; Duerig, U. Science 2015, 348 (6235), 679-684.

(13) Wang, W.; Dai, S.; Li, X.; Yang, J.; Srolovitz, D. J.; Zheng, Q. Nat. Commun. 2015, 6, 7853.

(14) Vahdat, A. S.; Cetinkaya, C. J. Appl. Phys. 2013, 114, 143502.

(15) Spanu, L.; Sorella, S.; Galli, G. Phys. Rev. Lett. 2009, 103 (19), 1-4. 
(16) Lebègue, S.; Harl, J.; Gould, T.; Ángyán, J. G.; Kresse, G.; Dobson, J. F. Phys. Rev. Lett. 2010, 105 (19), 196401.

(17) Björkman, T.; Gulans, A.; Krasheninnikov, A. V.; Nieminen, R. M. Phys. Rev. Lett. 2012, 108 (June), 1-5.

(18) Dobson, J. F.; Gould, T.; Vignale, G. Phys. Rev. X 2014, 4 (2), 1-9.

(19) Wang, J.; Sorescu, D. C.; Jeon, S.; Belianinov, A.; Kalinin, S. V; Baddorf, A. P.; Maksymovych, P. Nat. Commun. 2016, 7, 1-7.

(20) Li, P.; You, Z.; Cui, T. Sensors Actuators, A Phys. 2014, 217, 56-61.

(21) Ferguson, A.; Ca, I. T.; Backes, C.; Coleman, J. N.; Bergin, S. D. Chem. Mater. 2016, 28, $6355-6366$.

(22) Li, Z.; Wang, Y.; Kozbial, A.; Shenoy, G.; Zhou, F.; McGinley, R.; Ireland, P.; Morganstein, B.; Kunkel, A.; Surwade, S. P.; Li, L.; Liu, H. Nat. Mater. 2013, 12 (10), 925-931.

(23) Kozbial, A.; Trouba, C.; Liu, H.; Li, L. Langmuir 2017.

(24) Owens, D. K.; Wendt, R. C. J. Appl. Polym. Sci. 1969, 13, 1741-1747.

(25) Fowkes, F. M. J. Phys. Chem. 1962, 66 (2), 382.

(26) Ferrari, A. C.; Bonaccorso, F.; Falko, V.; Novoselov, K. S.; Roche, S.; Bøggild, P.; Borini, S.; Koppens, F.; Palermo, V.; Pugno, N.; Garrido, J. a.; Sordan, R.; Bianco, A.; Ballerini, L.; Prato, M.; Lidorikis, E.; Kivioja, J.; Marinelli, C.; Ryhänen, T.; Morpurgo, A.; Coleman, J. N.; Nicolosi, V.; Colombo, L.; Fert, A.; Garcia-Hernandez, M.; Bachtold, A.; Schneider, G. F.; Guinea, F.; Dekker, C.; Barbone, M.; Galiotis, C.; Grigorenko, A.; Konstantatos, G.; Kis, A.; Katsnelson, M.; Beenakker, C. W. J.; Vandersypen, L.; Loiseau, A.; Morandi, V.; Neumaier, D.; Treossi, E.; Pellegrini, V.; Polini, M.; 
Tredicucci, A.; Williams, G. M.; Hong, B. H.; Ahn, J. H.; Kim, J. M.; Zirath, H.; van Wees, B. J.; van der Zant, H.; Occhipinti, L.; Di Matteo, A.; Kinloch, I. a.; Seyller, T.; Quesnel, E.; Feng, X.; Teo, K.; Rupesinghe, N.; Hakonen, P.; Neil, S. R. T.; Tannock, Q.; Löfwander, T.; Kinaret, J. Nanoscale 2014, 7 (11), 4598-4810.

(27) Johnson, K. L. .; Kendall, K. .; Roberts, A. D. Proc. R. Soc. Lond. A 1971, 304, 301-313.

(28) McGuiggan, P. M.; Wallace, J. S.; Smith, D. T.; Sridhar, I.; Zheng, Z. W.; Johnson, K. L. J. Phys. D. Appl. Phys. 2007, 40 (19), 5984-5994.

(29) Christenson, H. K. Langmuir 1996, 12 (5), 1404-1405.

(30) Britton, J.; Cousens, N. E. A.; Coles, S. W.; Engers, C. D. Van; Babenko, V.; Murdock, A. T.; Koo, A.; Perkin, S.; Grobert, N. Langmuir 2014, 30, 11485-11492.

(31) Kumacheva, E.; Klein, J. J. Chem. Phys. 1998, 108 (16), 7010.

(32) Israelachvili, J. N. Intermolecular and Surface Forces, 3rd ed.; Elsevier: Kidlington, 2011.

(33) Perkin, S.; Chai, L.; Kampf, N.; Raviv, U.; Briscoe, W.; Dunlop, I.; Titmuss, S.; Seo, M.; Kumacheva, E.; Klein, J. Langmuir 2006, 22 (14), 6142-6152.

(34) Heuberger, M.; Luengo, G.; Israelachvili, J. Langmuir 1997, 13 (14), 3839-3848.

(35) Cheon, S.; Kihm, K. D.; Kim, H.; Lim, G.; Park, J. S.; Lee, J. S. Sci. Rep. 2014, 4, 6364.

(36) McPeak, K. M.; Jayanti, S. V; Kress, S. J. P.; Meyer, S.; Iotti, S.; Rossinelli, A.; Norris, D. J. ACS Photonics 2015, 2, 326-333.

(37) Paton, K. R.; Varrla, E.; Backes, C.; Smith, R. J.; Khan, U.; O’Neill, A.; Boland, C.; Lotya, M.; Istrate, O. M.; King, P.; Higgins, T.; Barwich, S.; May, P.; Puczkarski, P.; Ahmed, I.; Moebius, M.; Pettersson, H.; Long, E.; Coelho, J.; O’Brien, S. E.; McGuire, E. K.; Sanchez, B. M.; Duesberg, G. S.; McEvoy, N.; Pennycook, T. J.; Downing, C.; Crossley, A.; Nicolosi, V.; Coleman, J. N. Nat. Mater. 2014, 13 (6), 624-630. 
(38) Zeng, H.; Huang, J.; Tian, Y.; Li, L.; Tirrell, M. V; Israelachvili, J. N. Macromolecules 2016, 49, 5223-5231.

(39) Lin, S.; Shih, C. J.; Strano, M. S.; Blankschtein, D. J. Am. Chem. Soc. 2011, 133 (32), $12810-12823$.

(40) Huang, P. Y.; Ruiz-Vargas, C. S.; van der Zande, A. M.; Whitney, W. S.; Levendorf, M. P.; Kevek, J. W.; Garg, S.; Alden, J. S.; Hustedt, C. J.; Zhu, Y.; Park, J.; McEuen, P. L.; Muller, D. A. Nature 2011, 469 (7330), 389-392.

(41) Annett, J.; Cross, G. L. W. Nature 2016, 535 (7611), 271-275.

(42) Li, Z.; Kozbial, A.; Nioradze, N.; Parobek, D.; Shenoy, G. J.; Salim, M.; Amemiya, S.; Li, L.; Liu, H. ACS Nano 2016, 10 (1), 349-359.

(43) Klecha, E.; Arfaoui, I.; Richardi, J.; Pileni, M. Phys. Chem. Chem. Phys. 2011, 13, $2953-$ 2962.

(44) Ashraf, A.; Wu, Y.; Wang, M. C.; Aluru, N. R.; Dastgheib, S. A.; Nam, S. Langmuir 2014, 30, 12827-12836.

(45) Yang, S.; Kooij, E. S.; Poelsema, B.; Lohse, D.; Zandvliet, H. J. W. Europhys. Lett. 2008, 81. 


\section{FIGURES}

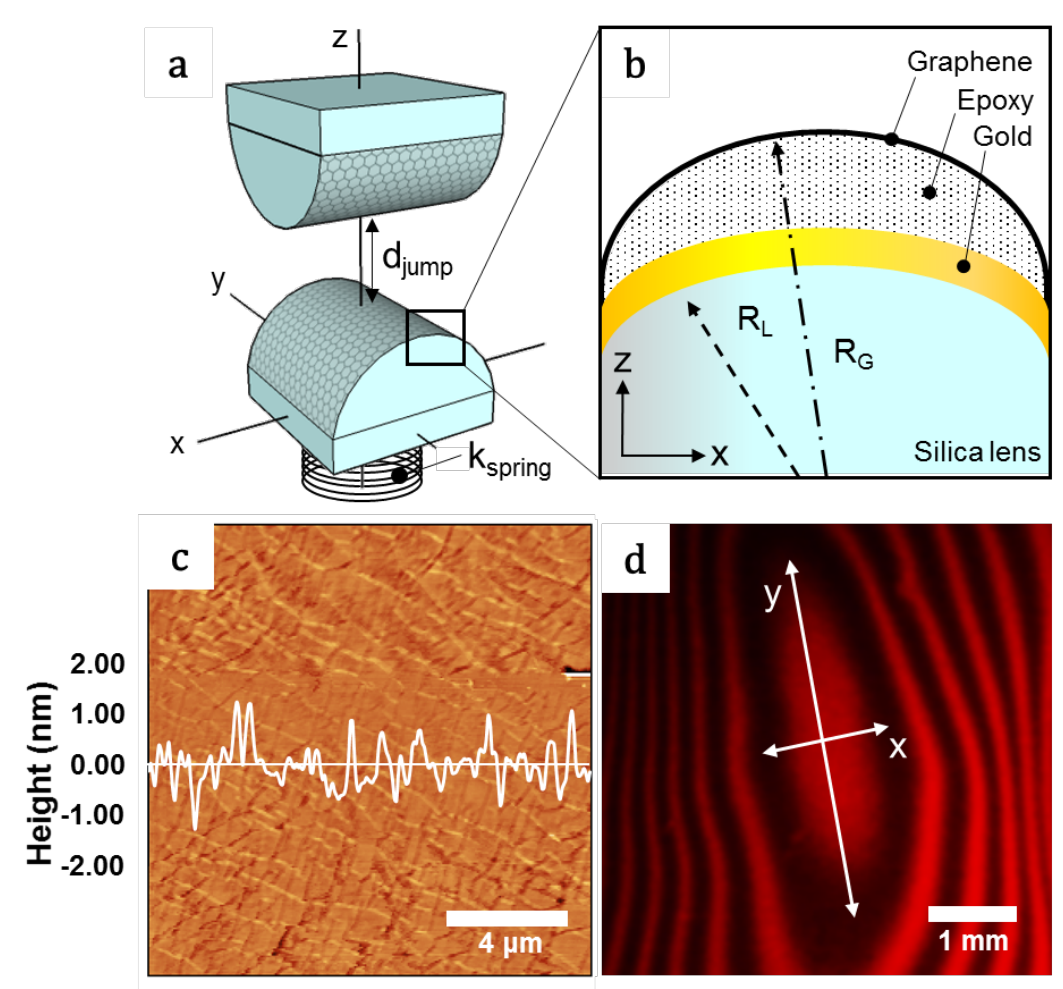

Figure 1. (a) Schematic illustration of the crossed-cylinder, single-contact geometry used for adhesion force measurements with the SFB. The surfaces are brought into adhesive contact and gradually pulled apart by a spring with known spring constant $\mathrm{k}_{\text {spring }}$ until they suddenly jump out of contact to a distance $\mathrm{d}_{\text {jump }}$. The pull-off force is calculated as $\mathrm{F}_{\text {adh }}=\mathrm{k}_{\text {spring }} \cdot \mathrm{d}_{\text {jump }}$. (b) Schematic of multilayer geometry consisting of a smooth sheet of SLG or FLG glued onto epoxy, supported by a gold-covered silica lens of curvature $R_{L}=10 \mathrm{~mm}$. Due to thickness inhomogeneity in the epoxy layer (exaggerated in the figure), the actual surface curvature $\left(R_{G}\right)$ differs from $R_{L}$. $(c)$ AFM height image. The inset shows the line profile over the line in the image. The RMSroughness is $0.7 \mathrm{~nm}$. (d) Optical image of Newton's rings for a prepared SFB lens viewed through a $560 \mathrm{~nm}$ filter. The $\mathrm{y}$-axis corresponds to the direction along the lens (y-axis shown in a). The bright/dark fringes correspond to locations of constructive/destructive interference. The 
height variation between consecutive bright fringes is $h=\lambda / 2 \mathrm{n}$, where $\lambda=560 \mathrm{~nm}$ and $\mathrm{n}=1.593$ is the glue refractive index, giving $\mathrm{h}=176 \mathrm{~nm}$ over a distance of $4 \mathrm{~mm}$ along the $\mathrm{y}$-axis of the lower lens in (a). 


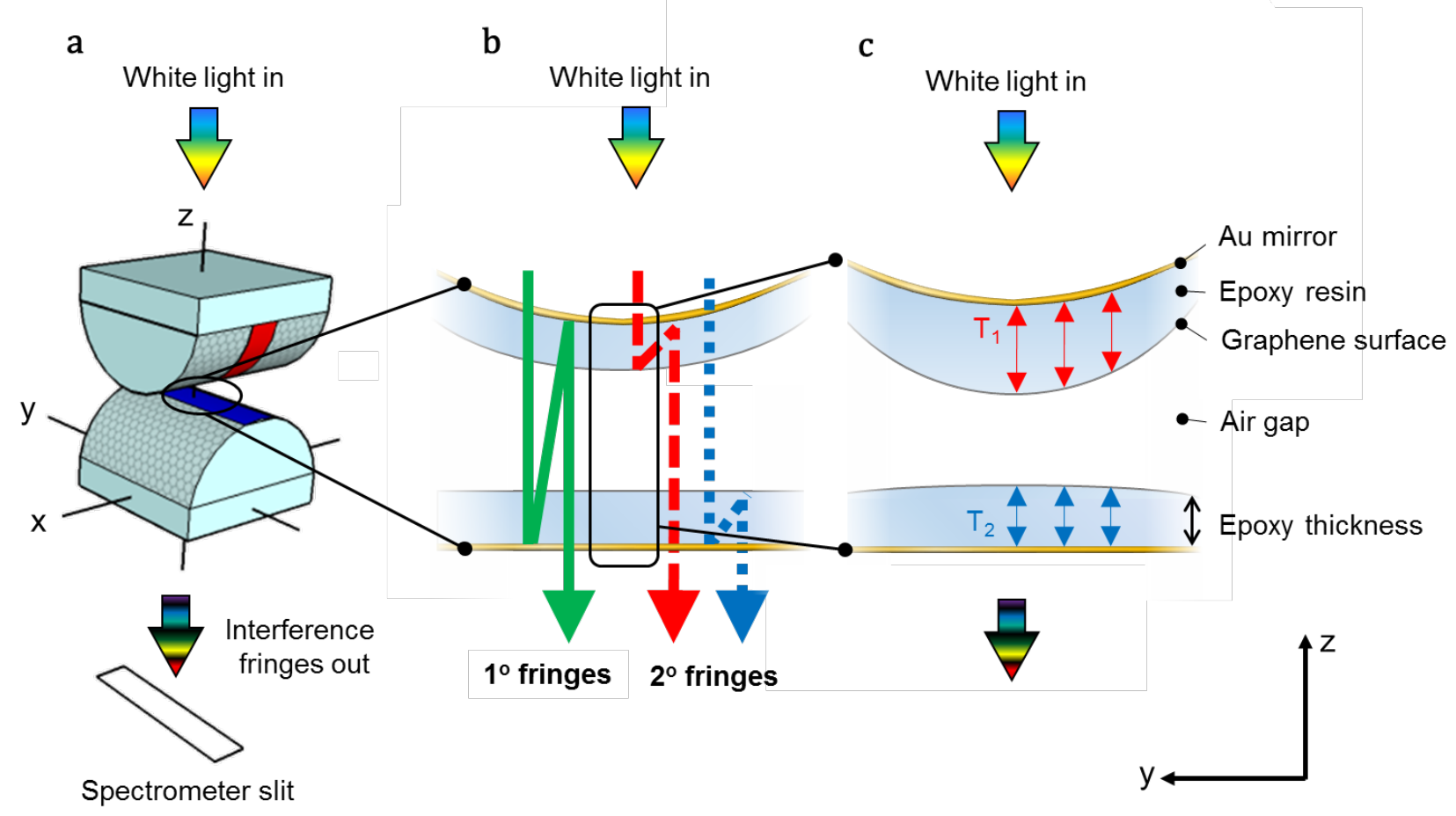

Figure 2. (a) Orientation of the surfaces relative to the spectrometer entrance slit. The ellipse indicates the contact position (b) Multiple reflections between the Au-mirrors give rise to primary fringes (green solid lines), whereas reflections inside the epoxy layers (i.e. between the Au mirror and the epoxy/graphene/air interface) produce secondary fringes (red dashed and blue dotted lines). (c) Exaggerated view of thickness inhomogeneities of the epoxy layers in the yz plane. The lower surface (blue band in a) has a negligible curvature and produces secondary fringes with large radius of curvature $R_{S / \mid}$ at the contact position, reflecting the large-scale random inhomogeneities of the layer thickness $T_{2}$ created by the layer fabrication method. The upper surface (red band in a) has a radius of curvature $R_{G}$ that is influenced by the inhomogeneity in thickness $T_{1}$ (i.e. $T_{1}=T_{1}(y)$ ). This inhomogeneity produces secondary fringes with curvature $R_{s}$. 

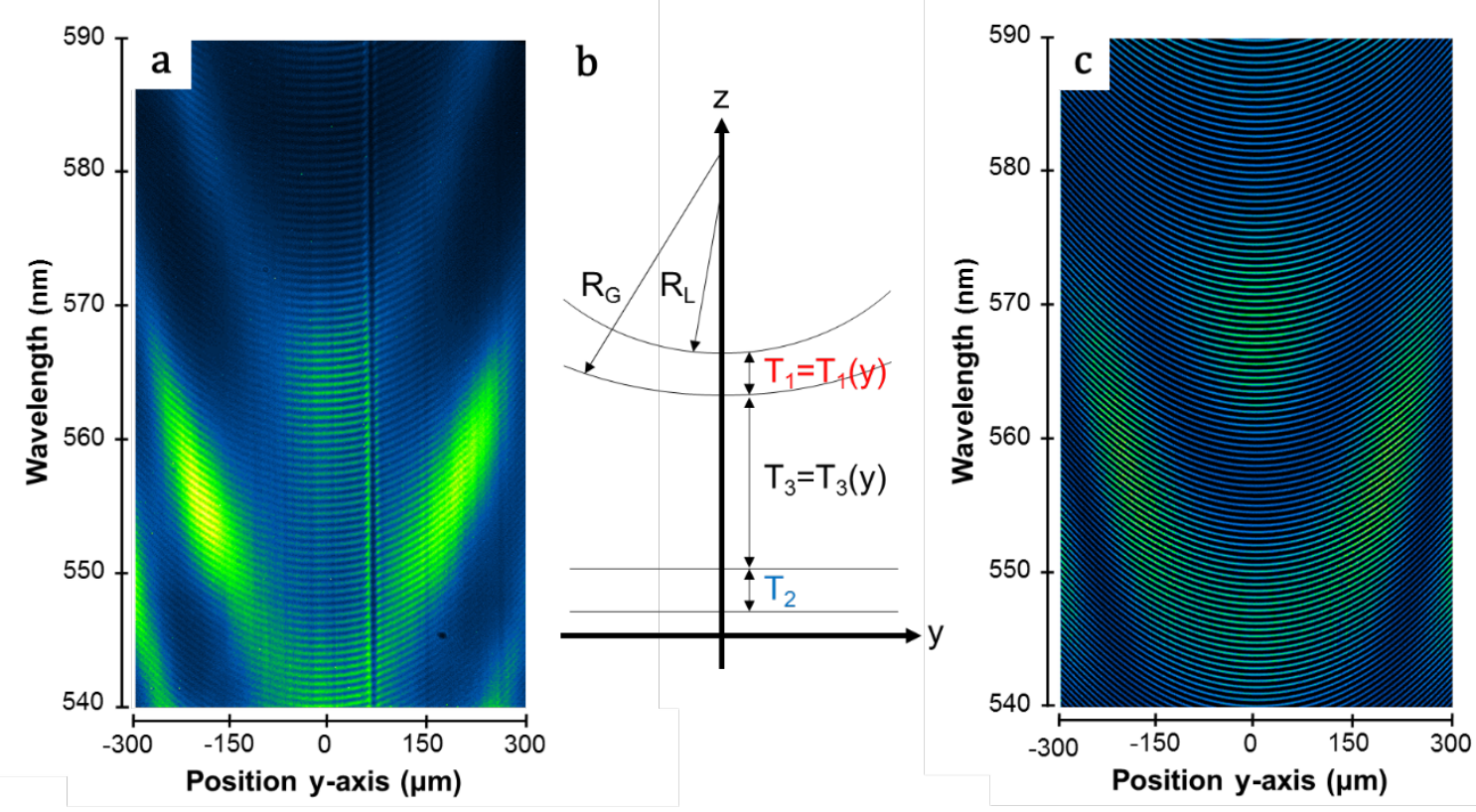

Figure 3. (a) Experimental interferogram. The closely spaced curved lines correspond to the primary fringes. The thin dark vertical line is produced by a speck of dust in the light path. The two large parabolic bands correspond to the secondary fringes used to determine $R_{S}$. The horizontal band at $550-560 \mathrm{~nm}$ is the secondary fringe corresponding to $R_{S / /}$ (b) Schematic showing the geometry used for the calculated interferogram. (c) Interferogram calculated using the value for $R_{S}$ measured from the experimental interferogram shown in (a). 

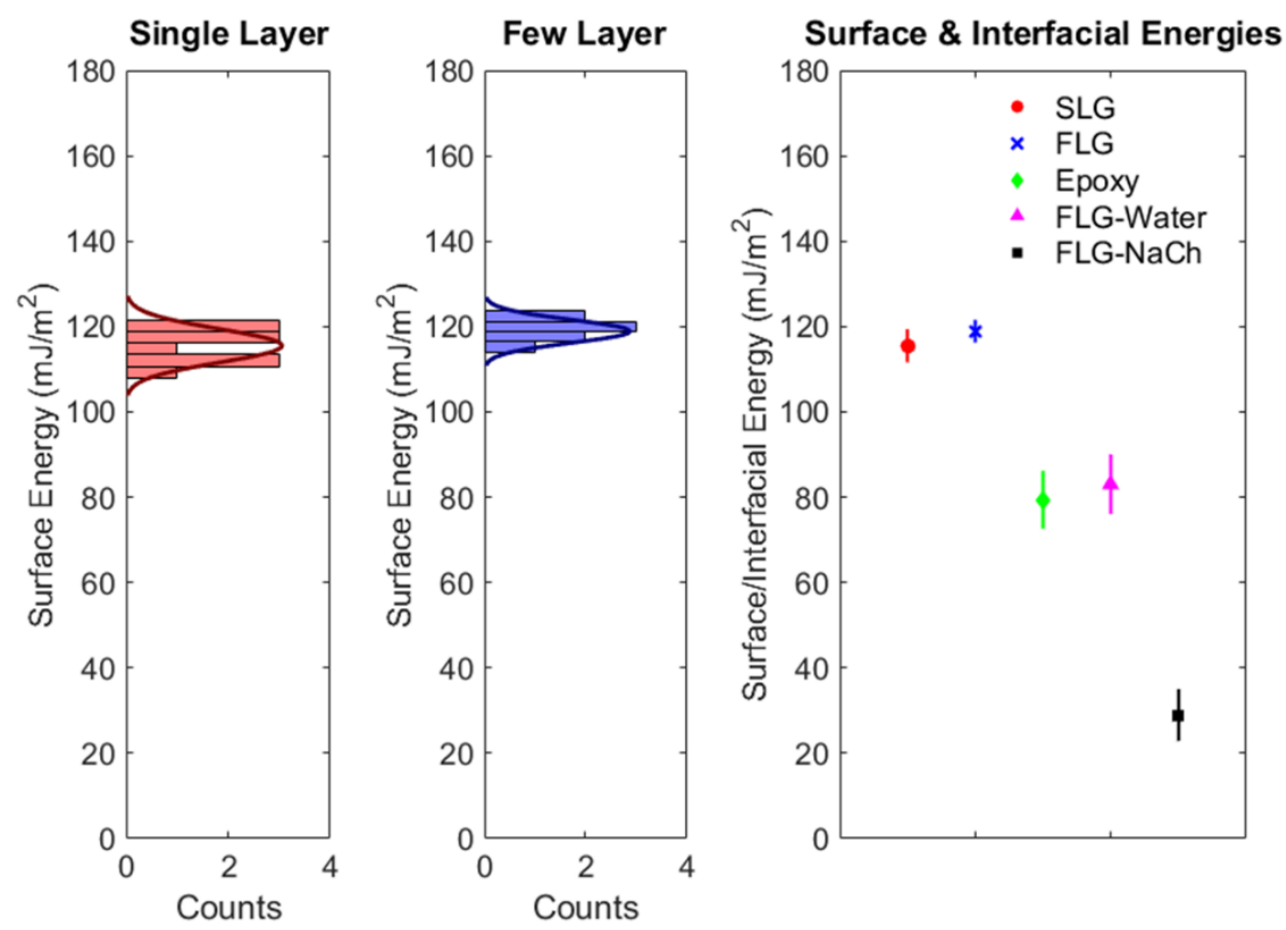

Figure 4. Overview of measured surface and interfacial energies. The histogram and probability distribution to determine the mean and standard deviation for SLG and FLG are shown on the left panels. The right panel shows all measured surface and interfacial energies. The surface energies of SLG, FLG and epoxy were measured in dry nitrogen. 


\section{TOC-Graphic}

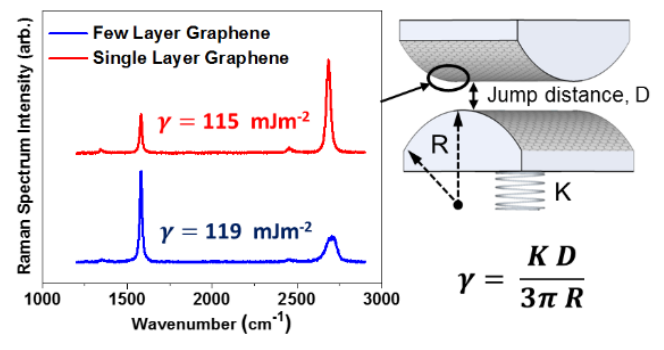

